\title{
TRANSVERSALITY THEOREMS ON GENERIC LINEARLY PERTURBED MAPPINGS
}

\author{
SHUNSUKE ICHIKI \\ In memory of John Mather
}

\begin{abstract}
In his celebrated paper "Generic projections", John Mather has given a striking transversality theorem and its applications on generic projections. On the other hand, in this paper, two transversality theorems on generic linearly perturbed $C^{r}$ mappings are shown $(r \geq 1)$. Moreover, some applications of the two theorems are also given.
\end{abstract}

\section{INTRODUCTION}

Throughout this paper, let $\ell, m$ and $n$ stand for positive integers. In this paper, unless otherwise stated, all manifolds and mappings are assumed to be of class $C^{r}(r \geq 1)$ and all manifolds are assumed to be without boundary and to have countable bases.

Let $F: U \rightarrow \mathbb{R}^{\ell}$ be a $C^{r}$ mapping from an open subset $U$ of $\mathbb{R}^{m}$. Then, for any linear mapping $\pi: \mathbb{R}^{m} \rightarrow \mathbb{R}^{\ell}$, set

$$
F_{\pi}=F+\pi .
$$

Here, the mapping $\pi$ in $F_{\pi}=F+\pi$ is restricted to $U$.

Let $\mathcal{L}\left(\mathbb{R}^{m}, \mathbb{R}^{\ell}\right)$ be the space consisting of all linear mappings of $\mathbb{R}^{m}$ into $\mathbb{R}^{\ell}$. Notice that we have the natural identification $\mathcal{L}\left(\mathbb{R}^{m}, \mathbb{R}^{\ell}\right)=\left(\mathbb{R}^{m}\right)^{\ell}$. By $N$, we denote a $C^{r}$ manifold of dimension $n$. For given $C^{r}$ mappings $f: N \rightarrow U$ and $F: U \rightarrow \mathbb{R}^{\ell}$, a property of mappings $F_{\pi} \circ f: N \rightarrow \mathbb{R}^{\ell}\left(\right.$ resp., $\left.\pi \circ f: N \rightarrow \mathbb{R}^{\ell}\right)$ will be said to be true for a generic linearly perturbed mapping (resp., a generic projection) if there exists a subset $\Sigma$ with Lebesgue measure zero of $\mathcal{L}\left(\mathbb{R}^{m}, \mathbb{R}^{\ell}\right)$ such that for any $\pi \in \mathcal{L}\left(\mathbb{R}^{m}, \mathbb{R}^{\ell}\right)-\Sigma$, the mapping $F_{\pi} \circ f: N \rightarrow \mathbb{R}^{\ell}$ (resp., $\pi \circ f: N \rightarrow \mathbb{R}^{\ell}$ ) has the property.

In his celebrated paper [5], for a given $C^{\infty}$ embedding $f: N \rightarrow \mathbb{R}^{m}$, John Mather has given a striking transversality theorem on a generic projection $\pi \circ f: N \rightarrow \mathbb{R}^{\ell}$ $\left(m>\ell\right.$ ), where $N$ is a $C^{\infty}$ manifold (for details on this result, see [5. Theorem 1 (p. 229)]). Moreover, in [5], as an application of this result, he has also shown that if $f: N \rightarrow \mathbb{R}^{m}$ is a $C^{\infty}$ embedding and $(n, \ell)$ is in the nice range of dimensions (for the definition of nice rage of dimensions, refer to [4]), then a generic projection $\pi \circ f: N \rightarrow \mathbb{R}^{\ell}(m>\ell)$ is stable, where $N$ is a compact $C^{\infty}$ manifold.

In [3], an improvement of the transversality theorem of [5] is given by replacing generic projections by generic linear perturbations. Namely, in $\underline{3}$, for a given $C^{\infty}$ embedding $f: N \rightarrow U$ and a given $C^{\infty}$ mapping $F: U \rightarrow \mathbb{R}^{\ell}$, a transversality

2010 Mathematics Subject Classification. 57R45, 57R42.

Key words and phrases. generic linear perturbation, transversality, immersion, injection.

Research Fellow PD of Japan Society for the Promotion of Science. 
theorem on a generic linearly perturbed mapping $F_{\pi} \circ f: N \rightarrow \mathbb{R}^{\ell}$ is given, where $N$ is a $C^{\infty}$ manifold and $\ell$ is an arbitrary positive integer which may possibly satisfy $m \leq \ell$.

Moreover, in 2], for a given $C^{\infty}$ immersion or a given $C^{\infty}$ injection $f: N \rightarrow U$, transversality theorems on a generic linearly perturbed mapping $F_{\pi} \circ f: N \rightarrow \mathbb{R}^{\ell}$ are given, where $N$ is a $C^{\infty}$ manifold, $F: U \rightarrow \mathbb{R}^{\ell}$ is a $C^{\infty}$ mapping and $\ell$ is an arbitrary positive integer which may possibly satisfy $m \leq \ell$.

On the other hand, in this paper, as improvements of some results in [2, two main transversality theorems (Theorems11and2 in Section2) and their applications on generic linearly perturbed mapping are given in the case where manifolds and mappings are not necessarily of class $C^{\infty}$.

The first main theorem (Theorem 10) is as follows. Let $f: N \rightarrow U$ (resp., $F: U \rightarrow \mathbb{R}^{\ell}$ ) be a $C^{r}$ immersion (resp., a $C^{r}$ mapping), where $N$ is a $C^{r}$ manifold (for the value of $r$, see Theorem 11). Then, generally, the composition $F \circ f$ does not necessarily yield a mapping transverse to the subfiber-bundle of the jet bundle $J^{1}\left(N, \mathbb{R}^{\ell}\right)$ with a fiber $\Sigma^{k}$, where $k$ is a positive integer satisfying $1 \leq k \leq \min \{n, \ell\}$ and

$$
\Sigma^{k}=\left\{j^{1} g(0) \in J^{1}(n, \ell) \mid \text { corank } J g(0)=k\right\} .
$$

Nevertheless, Theorem 1 asserts that a generic linearly perturbed mapping $F_{\pi} \circ f$ yields a mapping transverse to the subfiber-bundle of $J^{1}\left(N, \mathbb{R}^{\ell}\right)$ with $\Sigma^{k}$. The second main theorem (Theorem 2) is a specialized transversality theorem on crossings of a generic linearly perturbed mapping $F_{\pi} \circ f$, where $N$ is a $C^{r}$ manifold, $f: N \rightarrow U$ is a given $C^{r}$ injection and $F: U \rightarrow \mathbb{R}^{\ell}$ is a given $C^{r}$ mapping (for the value of $r$, see Theorem 21).

For a given $C^{2}$ immersion (resp., $C^{1}$ injection) $f: N \rightarrow U$ and a given $C^{2}$ mapping (resp., $C^{1}$ mapping) $F: U \rightarrow \mathbb{R}^{\ell}$, the following (1) and (2) (resp., (3)) are obtained as applications of Theorem 1 (resp., Theorem 2), where $N$ is a $C^{2}$ manifold (resp., a $C^{1}$ manifold).

(1) If $(n, \ell)=(n, 1)$, then a generic linearly perturbed function $F_{\pi} \circ f: N \rightarrow \mathbb{R}$ is a Morse function.

(2) If $\ell \geq 2 n$, then a generic linearly perturbed mapping $F_{\pi} \circ f: N \rightarrow \mathbb{R}^{\ell}$ is an immersion.

(3) If $\ell>2 n$, then a generic linearly perturbed mapping $F_{\pi} \circ f: N \rightarrow \mathbb{R}^{\ell}$ is an injection.

Furthermore, by combining the assertions (2) and (3), for a given $C^{2}$ embedding $f: N \rightarrow U$ and a given $C^{2}$ mapping $F: U \rightarrow \mathbb{R}^{\ell}$, we get the following assertion (4), where $N$ is a $C^{2}$ manifold.

(4) If $\ell>2 n$ and $N$ is compact, then a generic linearly perturbed mapping $F_{\pi} \circ f: N \rightarrow \mathbb{R}^{\ell}$ is an embedding.

In Section 2, some definitions are prepared, and the two main transversality theorems (Theorems 10 and 2) are stated. Section 3 (resp., Section 44) is devoted to the proof of Theorem 1 (resp., Theorem 2). In Section 15, the above assertions (1)-(4) are shown. In Section 6, the important lemma for the proofs of Theorems 1 and 2 (Lemma 1 in Section 2) is shown as an appendix. 


\section{Preliminaries and the statements of Theorems 1 and 2}

Firstly, the definition of transversality is given.

Definition 1. Let $N$ and $P$ be $C^{r}$ manifolds, and $Z$ be a $C^{r}$ submanifold of $P$ $(r \geq 1)$. Let $g: N \rightarrow P$ be a $C^{1}$ mapping.

(1) We say that $g: N \rightarrow P$ is transverse to $Z$ at $q$ if $g(q) \notin Z$ or in the case of $g(q) \in Z$, the following holds:

$$
d g_{q}\left(T_{q} N\right)+T_{g(q)} Z=T_{g(q)} P .
$$

(2) We say that $g: N \rightarrow P$ is transverse to $Z$ if for any $q \in N$, the mapping $g$ is transverse to $Z$ at $q$.

For the statement and the proof of Theorem [1. some definitions are prepared. Let $N$ be a $C^{r}$ manifold $(r \geq 2)$ and $J^{1}\left(N, \mathbb{R}^{\ell}\right)$ be the space of 1 -jets of mappings of $N$ into $\mathbb{R}^{\ell}$. Then, note that $J^{1}\left(N, \mathbb{R}^{\ell}\right)$ is a $C^{r-1}$ manifold. For a given $C^{r}$ mapping $g: N \rightarrow \mathbb{R}^{\ell}(r \geq 2)$, the mapping $j^{1} g: N \rightarrow J^{1}\left(N, \mathbb{R}^{\ell}\right)$ is defined by $q \mapsto j^{1} g(q)$. Then, notice that the mapping $j^{1} g: N \rightarrow J^{1}\left(N, \mathbb{R}^{\ell}\right)$ is of class $C^{r-1}$. For details on the space $J^{1}\left(N, \mathbb{R}^{\ell}\right)$ or the mapping $j^{1} g: N \rightarrow J^{r}\left(N, \mathbb{R}^{\ell}\right)$, see for example, [1.

Now, let $\left\{\left(U_{\lambda}, \varphi_{\lambda}\right)\right\}_{\lambda \in \Lambda}$ be a coordinate neighborhood system of $N$. Let $\Pi$ : $J^{1}\left(N, \mathbb{R}^{\ell}\right) \rightarrow N \times \mathbb{R}^{\ell}$ be the natural projection defined by $\Pi\left(j^{1} g(q)\right)=(q, g(q))$. Let $\Phi_{\lambda}: \Pi^{-1}\left(U_{\lambda} \times \mathbb{R}^{\ell}\right) \rightarrow \varphi_{\lambda}\left(U_{\lambda}\right) \times \mathbb{R}^{\ell} \times J^{1}(n, \ell)$ be the homeomorphism defined by

$$
\Phi_{\lambda}\left(j^{1} g(q)\right)=\left(\varphi_{\lambda}(q), g(q), j^{1}\left(\psi_{\lambda} \circ g \circ \varphi_{\lambda}^{-1} \circ \widetilde{\varphi}_{\lambda}\right)(0)\right),
$$

where $J^{1}(n, \ell)=\left\{j^{1} g(0) \mid g:\left(\mathbb{R}^{n}, 0\right) \rightarrow\left(\mathbb{R}^{\ell}, 0\right)\right\}$ and $\widetilde{\varphi}_{\lambda}: \mathbb{R}^{n} \rightarrow \mathbb{R}^{n}$ (resp., $\psi_{\lambda}: \mathbb{R}^{m} \rightarrow \mathbb{R}^{m}$ ) is the translation given by $\widetilde{\varphi}_{\lambda}(0)=\varphi_{\lambda}(q)$ (resp., $\psi_{\lambda}(g(q))=0$ ). Then, $\left\{\left(\Pi^{-1}\left(U_{\lambda} \times \mathbb{R}^{\ell}\right), \Phi_{\lambda}\right)\right\}_{\lambda \in \Lambda}$ is a coordinate neighborhood system of $J^{1}\left(N, \mathbb{R}^{\ell}\right)$. Set

$$
\Sigma^{k}=\left\{j^{1} g(0) \in J^{1}(n, \ell) \mid \text { corank } J g(0)=k\right\},
$$

where corank $J g(0)=\min \{n, \ell\}-\operatorname{rank} J g(0)$ and $k=1,2, \ldots, \min \{n, \ell\}$. Set

$$
\Sigma^{k}\left(N, \mathbb{R}^{\ell}\right)=\bigcup_{\lambda \in \Lambda} \Phi_{\lambda}^{-1}\left(\varphi_{\lambda}\left(U_{\lambda}\right) \times \mathbb{R}^{\ell} \times \Sigma^{k}\right)
$$

Then, the set $\Sigma^{k}\left(N, \mathbb{R}^{\ell}\right)$ is a submanifold of $J^{1}\left(N, \mathbb{R}^{\ell}\right)$ satisfying

$$
\begin{aligned}
\operatorname{codim} \Sigma^{k}\left(N, \mathbb{R}^{\ell}\right) & =\operatorname{dim} J^{1}\left(N, \mathbb{R}^{\ell}\right)-\operatorname{dim} \Sigma^{k}\left(N, \mathbb{R}^{\ell}\right) \\
& =(n-v+k)(\ell-v+k),
\end{aligned}
$$

where $v=\min \{n, \ell\}$. (For details on $\Sigma^{k}$ and $\Sigma^{k}\left(N, \mathbb{R}^{\ell}\right)$, see for instance [1, pp. 60-61).

Then, the first main theorem in this paper is the following.

Theorem 1. Let $f$ be a $C^{r}$ immersion of $N$ into an open subset $U$ of $\mathbb{R}^{m}$, where $N$ is a $C^{r}$ manifold of dimension $n$. Let $F: U \rightarrow \mathbb{R}^{\ell}$ be a $C^{r}$ mapping and $k$ be a positive integer satisfying $1 \leq k \leq \min \{n, \ell\}$. If

$$
r>\max \left\{\operatorname{dim} N-\operatorname{codim} \Sigma^{k}\left(N, \mathbb{R}^{\ell}\right), 0\right\}+1,
$$

then there exists a subset $\Sigma$ with Lebesgue measure zero of $\mathcal{L}\left(\mathbb{R}^{m}, \mathbb{R}^{\ell}\right)$ such that for any $\pi \in \mathcal{L}\left(\mathbb{R}^{m}, \mathbb{R}^{\ell}\right)-\Sigma$, the mapping $j^{1}\left(F_{\pi} \circ f\right): N \rightarrow J^{1}\left(N, \mathbb{R}^{\ell}\right)$ is transverse to the submanifold $\Sigma^{k}\left(N, \mathbb{R}^{\ell}\right)$. 
Now, in order to state the second main theorem (Theorem 2), we will prepare some definitions. Let $N$ be a $C^{r}$ manifold $(r \geq 1)$. Set

$$
N^{(s)}=\left\{\left(q_{1}, q_{2}, \ldots, q_{s}\right) \in N^{s} \mid q_{i} \neq q_{j}(i \neq j)\right\} .
$$

Note that $N^{(s)}$ is an open submanifold of $N^{s}$. For any mapping $g: N \rightarrow \mathbb{R}^{\ell}$, let $g^{(s)}: N^{(s)} \rightarrow\left(\mathbb{R}^{\ell}\right)^{s}$ be the mapping given by

$$
g^{(s)}\left(q_{1}, q_{2}, \ldots, q_{s}\right)=\left(g\left(q_{1}\right), g\left(q_{2}\right), \ldots, g\left(q_{s}\right)\right) .
$$

Set $\Delta_{s}=\left\{(y, \ldots, y) \in\left(\mathbb{R}^{\ell}\right)^{s} \mid y \in \mathbb{R}^{\ell}\right\}$. Then, $\Delta_{s}$ is a submanifold of $\left(\mathbb{R}^{\ell}\right)^{s}$ satisfying

$$
\operatorname{codim} \Delta_{s}=\operatorname{dim}\left(\mathbb{R}^{\ell}\right)^{s}-\operatorname{dim} \Delta_{s}=\ell(s-1) .
$$

Definition 2. Let $g$ be a $C^{1}$ mapping of $N$ into $\mathbb{R}^{\ell}$, where $N$ is a $C^{r}$ manifold $(r \geq 1)$. Then, $g$ is called a mapping with normal crossings if for any positive integer $s(s \geq 2)$, the mapping $g^{(s)}: N^{(s)} \rightarrow\left(\mathbb{R}^{\ell}\right)^{s}$ is transverse to $\Delta_{s}$.

As in [2, for any injection $f: N \rightarrow \mathbb{R}^{m}$, set

$$
s_{f}=\max \left\{s \mid \forall\left(q_{1}, q_{2}, \ldots, q_{s}\right) \in N^{(s)}, \operatorname{dim} \sum_{i=2}^{s} \mathbb{R} \overrightarrow{f\left(q_{1}\right) f\left(q_{i}\right)}=s-1\right\} .
$$

Since the mapping $f$ is an injection, we have $2 \leq s_{f}$. Since $f\left(q_{1}\right), f\left(q_{2}\right), \ldots, f\left(q_{s_{f}}\right)$ are points of $\mathbb{R}^{m}$, it follows that $s_{f} \leq m+1$. Hence, we get

$$
2 \leq s_{f} \leq m+1 \text {. }
$$

Moreover, in the following, for a set $X$, we denote the number of its elements (or its cardinality) by $|X|$. Then, the second main theorem in this paper is the following.

Theorem 2. Let $f$ be a $C^{r}$ injection of $N$ into an open subset $U$ of $\mathbb{R}^{m}$, where $N$ is a $C^{r}$ manifold of dimension $n$. Let $F: U \rightarrow \mathbb{R}^{\ell}$ be a $C^{r}$ mapping. If

$$
r>\max \left\{s_{0}, 0\right\},
$$

then there exists a subset $\Sigma$ of $\mathcal{L}\left(\mathbb{R}^{m}, \mathbb{R}^{\ell}\right)$ with Lebesgue measure zero such that for any $\pi \in \mathcal{L}\left(\mathbb{R}^{m}, \mathbb{R}^{\ell}\right)-\Sigma$, and for any $s\left(2 \leq s \leq s_{f}\right)$, the $C^{r}$ mapping $\left(F_{\pi} \circ f\right)^{(s)}$ : $N^{(s)} \rightarrow\left(\mathbb{R}^{\ell}\right)^{s}$ is transverse to the submanifold $\Delta_{s}$, where

$$
s_{0}=\max \left\{s(n-\ell)+\ell \mid 2 \leq s \leq s_{f}\right\} .
$$

Moreover, if the mapping $F_{\pi}$ satisfies that $\left|F_{\pi}^{-1}(y)\right| \leq s_{f}$ for any $y \in \mathbb{R}^{\ell}$, then $F_{\pi} \circ f: N \rightarrow \mathbb{R}^{\ell}$ is a $C^{r}$ mapping with normal crossings.

Remark 1. (1) There is an advantage that the domain of the mapping $F$ is an arbitrary open set. Suppose that $U=\mathbb{R}$. Let $F: \mathbb{R} \rightarrow \mathbb{R}$ be the function defined by $x \mapsto|x|$. Since $F$ is not differentiable at $x=0$, we cannot apply Theorems 1 and 2 to $F: \mathbb{R} \rightarrow \mathbb{R}$.

On the other hand, if $U=\mathbb{R}-\{0\}$, then Theorems 1 and 2 can be applied to the restriction $\left.F\right|_{U}$.

(2) As in 2, there is a case of $s_{f}=3$ as follows. If $n+1 \leq m, N=S^{n}$ and $f: S^{n} \rightarrow \mathbb{R}^{m}$ is the inclusion $f(x)=(x, 0, \ldots, 0)$, then we get $s_{f}=3$. Indeed, suppose that there exists a point $\left(q_{1}, q_{2}, q_{3}\right) \in\left(S^{n}\right)^{(3)}$ satisfying $\operatorname{dim} \sum_{i=2}^{3} \mathbb{R} \overrightarrow{f\left(q_{1}\right) f\left(q_{i}\right)}=1$. Then, since the number of the intersections of $f\left(S^{n}\right)$ and a straight line of $\mathbb{R}^{m}$ is at most two, this contradicts the 
TRANSVERSALITY THEOREMS ON GENERIC LINEARLY PERTURBED MAPPINGS

assumption. Thus, we have $s_{f} \geq 3$. From $S^{1} \times\{0\} \subset f\left(S^{n}\right)$, we get $s_{f}<4$, where $0=\underbrace{(0, \ldots, 0)}_{(m-2) \text {-tuple }}$. Therefore, it follows that $s_{f}=3$.

(3) The essential idea for the proofs of Theorems 1 and 2 is to apply Lemma 1 and it is similar to the idea of the proofs of [2, Theorems 1 and 2]. Note that in the special case $r=\infty$, from some results in 2, the results in this paper (Theorems 1 and 2 in this section and Corollaries 1 to 7 in Section 5) can be obtained.

The following well known result is important for the proofs of Theorems 1 and 2 In [1, the proof of Lemma 1 in the case $r=\infty$ is given. Hence, for the sake of readers' convenience, the proof of Lemma 1 is given in Section 6 as an appendix.

Lemma 1 ([1]). Let $N, A, P$ be $C^{r}$ manifolds, $Z$ be a $C^{r}$ submanifold of $P$ and $\Gamma: N \times A \rightarrow P$ be a $C^{r}$ mapping. If

$$
r>\max \{\operatorname{dim} N-\operatorname{codim} Z, 0\},
$$

and $\Gamma$ is transverse to $Z$, then there exists a subset $\Sigma$ of $A$ with Lebesgue measure zero such that for any $a \in A-\Sigma$, the $C^{r}$ mapping $\Gamma_{a}: N \rightarrow P$ is transverse to $Z$, where $\operatorname{codim} Z=\operatorname{dim} P-\operatorname{dim} Z$ and $\Gamma_{a}(q)=\Gamma(q, a)$.

\section{Proof of Theorem 1}

In this proof, for a positive integer $\widetilde{n}$, we denote the $\widetilde{n} \times \widetilde{n}$ unit matrix by $E_{\widetilde{n}}$. Let $\left(\alpha_{i j}\right)_{1 \leq i \leq \ell, 1 \leq j \leq m}$ be a representing matrix of a linear mapping $\pi: \mathbb{R}^{m} \rightarrow \mathbb{R}^{\ell}$. Set $F_{\alpha}=F_{\pi}$. Then, we have

$$
F_{\alpha}(x)=\left(F_{1}(x)+\sum_{j=1}^{m} \alpha_{1 j} x_{j}, F_{2}(x)+\sum_{j=1}^{m} \alpha_{2 j} x_{j}, \ldots, F_{\ell}(x)+\sum_{j=1}^{m} \alpha_{\ell j} x_{j}\right),
$$

where $F=\left(F_{1}, F_{2}, \ldots, F_{\ell}\right), \alpha=\left(\alpha_{11}, \alpha_{12}, \ldots, \alpha_{1 m}, \ldots, \alpha_{\ell 1}, \alpha_{\ell 2}, \ldots, \alpha_{\ell m}\right) \in\left(\mathbb{R}^{m}\right)^{\ell}$ and $x=\left(x_{1}, x_{2}, \ldots, x_{m}\right)$. For a given $C^{r}$ immersion $f: N \rightarrow U$, the $C^{r}$ mapping $F_{\alpha} \circ f: N \rightarrow \mathbb{R}^{\ell}$ is given as follows:

$$
F_{\alpha} \circ f=\left(F_{1} \circ f+\sum_{j=1}^{m} \alpha_{1 j} f_{j}, F_{2} \circ f+\sum_{j=1}^{m} \alpha_{2 j} f_{j}, \ldots, F_{\ell} \circ f+\sum_{j=1}^{m} \alpha_{\ell j} f_{j}\right),
$$

where $f=\left(f_{1}, f_{2}, \ldots, f_{m}\right)$. Since we have the natural identification $\mathcal{L}\left(\mathbb{R}^{m}, \mathbb{R}^{\ell}\right)=$ $\left(\mathbb{R}^{m}\right)^{\ell}$, for the proof, it is sufficient to show that there exists a subset $\Sigma$ with Lebesgue measure zero of $\left(\mathbb{R}^{m}\right)^{\ell}$ such that for any $\alpha \in\left(\mathbb{R}^{m}\right)^{\ell}-\Sigma$, the mapping $j^{1}\left(F_{\alpha} \circ f\right): N \rightarrow J^{1}\left(N, \mathbb{R}^{\ell}\right)$ is transverse to $\Sigma^{k}\left(N, \mathbb{R}^{\ell}\right)$.

Now, let $\Gamma: N \times\left(\mathbb{R}^{m}\right)^{\ell} \rightarrow J^{1}\left(N, \mathbb{R}^{\ell}\right)$ be the $C^{r-1}$ mapping defined by

$$
\Gamma(q, \alpha)=j^{1}\left(F_{\alpha} \circ f\right)(q) .
$$

Note that $r-1>\max \left\{\operatorname{dim} N-\operatorname{codim} \Sigma^{k}\left(N, \mathbb{R}^{\ell}\right), 0\right\}$. Thus, if $\Gamma$ is transverse to $\Sigma^{k}\left(N, \mathbb{R}^{\ell}\right)$, then from Lemma 1 there exists a subset $\Sigma$ of $\left(\mathbb{R}^{m}\right)^{\ell}$ with Lebesgue measure zero such that for any $\alpha \in\left(\mathbb{R}^{m}\right)^{\ell}-\Sigma$, the $C^{r-1}$ mapping $\Gamma_{\alpha}: N \rightarrow$ $J^{1}\left(N, \mathbb{R}^{\ell}\right)\left(\Gamma_{\alpha}=j^{1}\left(F_{\alpha} \circ f\right)\right)$ is transverse to $\Sigma^{k}\left(N, \mathbb{R}^{\ell}\right)$. Therefore, for the proof, it is sufficient to show that if $\Gamma(\widetilde{q}, \widetilde{\alpha}) \in \Sigma^{k}\left(N, \mathbb{R}^{\ell}\right)$, then the following holds:

$$
d \Gamma_{(\widetilde{q}, \widetilde{\alpha})}\left(T_{(\widetilde{q}, \widetilde{\alpha})}\left(N \times\left(\mathbb{R}^{m}\right)^{\ell}\right)\right)+T_{\Gamma(\widetilde{q}, \widetilde{\alpha})} \Sigma^{k}\left(N, \mathbb{R}^{\ell}\right)=T_{\Gamma(\widetilde{q}, \widetilde{\alpha})} J^{1}\left(N, \mathbb{R}^{\ell}\right) .
$$


As in Section 2 let $\left\{\left(U_{\lambda}, \varphi_{\lambda}\right)\right\}_{\lambda \in \Lambda}$ (resp., $\left.\left\{\left(\Pi^{-1}\left(U_{\lambda} \times \mathbb{R}^{\ell}\right), \Phi_{\lambda}\right)\right\}_{\lambda \in \Lambda}\right)$ be a coordinate neighborhood system of $N$ (resp., $\left.J^{1}\left(N, \mathbb{R}^{\ell}\right)\right)$. There exists a coordinate neighbor$\operatorname{hood}\left(U_{\tilde{\lambda}} \times\left(\mathbb{R}^{m}\right)^{\ell}, \varphi_{\tilde{\lambda}} \times i d\right)$ containing the point $(\widetilde{q}, \widetilde{\alpha})$ of $N \times\left(\mathbb{R}^{m}\right)^{\ell}$, where id is the identity mapping of $\left(\mathbb{R}^{m}\right)^{\ell}$ into $\left(\mathbb{R}^{m}\right)^{\ell}$, and the mapping $\varphi_{\tilde{\lambda}} \times i d: U_{\tilde{\lambda}} \times\left(\mathbb{R}^{m}\right)^{\ell} \rightarrow$ $\varphi_{\tilde{\lambda}}\left(U_{\tilde{\lambda}}\right) \times\left(\mathbb{R}^{m}\right)^{\ell}\left(\subset \mathbb{R}^{n} \times\left(\mathbb{R}^{m}\right)^{\ell}\right)$ is given by $\left(\varphi_{\tilde{\lambda}} \times i d\right)(q, \alpha)=\left(\varphi_{\tilde{\lambda}}(q), i d(\alpha)\right)$. There exists a coordinate neighborhood $\left(\Pi^{-1}\left(U_{\widetilde{\lambda}} \times \mathbb{R}^{\ell}\right), \Phi_{\tilde{\lambda}}\right)$ containing the point $\Gamma(\widetilde{q}, \widetilde{\alpha})$ of $J^{1}\left(N, \mathbb{R}^{\ell}\right)$. Let $t=\left(t_{1}, t_{2}, \ldots, t_{n}\right) \in \mathbb{R}^{n}$ be a local coordinate on $\varphi_{\tilde{\lambda}}\left(U_{\tilde{\lambda}}\right)$ containing $\varphi_{\widetilde{\lambda}}(\widetilde{q})$. Then, the mapping $\Gamma$ is locally given by the following:

$$
\begin{aligned}
& \left(\Phi_{\tilde{\lambda}} \circ \Gamma \circ\left(\varphi_{\tilde{\lambda}} \times i d\right)^{-1}\right)(t, \alpha) \\
& =\left(\Phi_{\tilde{\lambda}} \circ j^{1}\left(F_{\alpha} \circ f\right) \circ \varphi_{\tilde{\lambda}}^{-1}\right)(t) \\
& =\left(t,\left(F_{\alpha} \circ f \circ \varphi_{\tilde{\lambda}}^{-1}\right)(t),\right. \\
& \frac{\partial\left(F_{\alpha, 1} \circ f \circ \varphi_{\tilde{\lambda}}^{-1}\right)}{\partial t_{1}}(t), \frac{\partial\left(F_{\alpha, 1} \circ f \circ \varphi_{\tilde{\lambda}}^{-1}\right)}{\partial t_{2}}(t), \ldots, \frac{\partial\left(F_{\alpha, 1} \circ f \circ \varphi_{\tilde{\lambda}}^{-1}\right)}{\partial t_{n}}(t), \\
& \frac{\partial\left(F_{\alpha, 2} \circ f \circ \varphi_{\tilde{\lambda}}^{-1}\right)}{\partial t_{1}}(t), \frac{\partial\left(F_{\alpha, 2} \circ f \circ \varphi_{\tilde{\lambda}}^{-1}\right)}{\partial t_{2}}(t), \ldots, \frac{\partial\left(F_{\alpha, 2} \circ f \circ \varphi_{\tilde{\lambda}}^{-1}\right)}{\partial t_{n}}(t), \\
& \text {....... } \\
& \left.\frac{\partial\left(F_{\alpha, \ell} \circ f \circ \varphi_{\tilde{\lambda}}^{-1}\right)}{\partial t_{1}}(t), \frac{\partial\left(F_{\alpha, \ell} \circ f \circ \varphi_{\tilde{\lambda}}^{-1}\right)}{\partial t_{2}}(t), \ldots, \frac{\partial\left(F_{\alpha, \ell} \circ f \circ \varphi_{\tilde{\lambda}}^{-1}\right)}{\partial t_{n}}(t)\right) \\
& =\left(t,\left(F_{\alpha} \circ f \circ \varphi_{\tilde{\lambda}}^{-1}\right)(t),\right. \\
& \frac{\partial F_{1} \circ \tilde{f}}{\partial t_{1}}(t)+\sum_{j=1}^{m} \alpha_{1 j} \frac{\partial \tilde{f}_{j}}{\partial t_{1}}(t), \frac{\partial F_{1} \circ \tilde{f}}{\partial t_{2}}(t)+\sum_{j=1}^{m} \alpha_{1 j} \frac{\partial \tilde{f}_{j}}{\partial t_{2}}(t), \ldots, \frac{\partial F_{1} \circ \tilde{f}}{\partial t_{n}}(t)+\sum_{j=1}^{m} \alpha_{1 j} \frac{\partial \tilde{f}_{j}}{\partial t_{n}}(t), \\
& \frac{\partial F_{2} \circ \tilde{f}}{\partial t_{1}}(t)+\sum_{j=1}^{m} \alpha_{2 j} \frac{\partial \tilde{f}_{j}}{\partial t_{1}}(t), \frac{\partial F_{2} \circ \tilde{f}}{\partial t_{2}}(t)+\sum_{j=1}^{m} \alpha_{2 j} \frac{\partial \tilde{f}_{j}}{\partial t_{2}}(t), \ldots, \frac{\partial F_{2} \circ \tilde{f}}{\partial t_{n}}(t)+\sum_{j=1}^{m} \alpha_{2 j} \frac{\partial \tilde{f}_{j}}{\partial t_{n}}(t), \\
& \left.\frac{\partial F_{\ell} \circ \tilde{f}}{\partial t_{1}}(t)+\sum_{j=1}^{m} \alpha_{\ell j} \frac{\partial \tilde{f}_{j}}{\partial t_{1}}(t), \frac{\partial F_{\ell} \circ \tilde{f}}{\partial t_{2}}(t)+\sum_{j=1}^{m} \alpha_{\ell j} \frac{\partial \tilde{f}_{j}}{\partial t_{2}}(t), \ldots, \frac{\partial F_{\ell} \circ \tilde{f}}{\partial t_{n}}(t)+\sum_{j=1}^{m} \alpha_{\ell j} \frac{\partial \tilde{f}_{j}}{\partial t_{n}}(t)\right),
\end{aligned}
$$

where $F_{\alpha}=\left(F_{\alpha, 1}, F_{\alpha, 2}, \ldots, F_{\alpha, \ell}\right)$ and $\widetilde{f}=\left(\widetilde{f}_{1}, \widetilde{f}_{2}, \ldots, \widetilde{f}_{m}\right)=\left(f_{1} \circ \varphi_{\tilde{\lambda}}^{-1}, f_{2} \circ \varphi_{\tilde{\lambda}}^{-1}, \ldots, f_{m} \circ\right.$ $\left.\varphi_{\tilde{\lambda}}^{-1}\right)=f \circ \varphi_{\tilde{\lambda}}^{-1}$. The Jacobian matrix of $\Gamma$ at $(\widetilde{q}, \widetilde{\alpha})$ is the following:

$$
J \Gamma_{(\widetilde{q}, \widetilde{\alpha})}=\left(\begin{array}{c|cccc}
E_{n} & 0 & \cdots & \cdots & 0 \\
\hline & * & \cdots & \cdots & * \\
& { }^{t}\left(J f_{\widetilde{q}}\right) & & 0 & \\
& & t\left(J f_{\widetilde{q}}\right) & & \\
& & 0 & \ddots & \\
& & & & t\left(J f_{\widetilde{q}}\right)
\end{array}\right)_{(t, \alpha)=\left(\varphi_{\widetilde{\lambda}}(\widetilde{q}), \widetilde{\alpha}\right)},
$$

where $J f_{\widetilde{q}}$ is the Jacobian matrix of $f$ at $\widetilde{q}$. Notice that ${ }^{t}\left(J f_{\widetilde{q}}\right)$ is the transpose of $J f_{\widetilde{q}}$ and that there are $\ell$ copies of ${ }^{t}\left(J f_{\widetilde{q}}\right)$ in the above description of $J \Gamma_{(\widetilde{q}, \widetilde{\alpha})}$. Since $\Sigma^{k}\left(N, \mathbb{R}^{\ell}\right)$ is a subfiber-bundle of $J^{1}\left(N, \mathbb{R}^{\ell}\right)$ with the fiber $\Sigma^{k}$, in order to show 
(3.3), it is sufficient to prove that the matrix $M_{1}$ given below has rank $n+\ell+n \ell$ :

$$
M_{1}=\left(\begin{array}{c|cccc}
E_{n+\ell} & * & \ldots & \cdots & * \\
\hline \multirow{2}{*}{0} & { }^{t}\left(J f_{\widetilde{q}}\right) & t_{(}\left(J f_{\widetilde{q}}\right) & 0 & \\
& & 0 & \ddots & \\
& & & & { }^{t}\left(J f_{\widetilde{q}}\right)
\end{array}\right)_{(t, \alpha)=\left(\varphi_{\tilde{\lambda}}(\widetilde{q}), \widetilde{\alpha}\right)} .
$$

Notice that there are $\ell$ copies of ${ }^{t}\left(J f_{\widetilde{q}}\right)$ in the above description of $M_{1}$. Note that for any $i(1 \leq i \leq m \ell)$, the $(n+\ell+i)$-th column vector of $M_{1}$ coincides with the $(n+i)$-th column vector of $J \Gamma_{(\widetilde{q}, \widetilde{\alpha})}$. Since $f$ is an immersion $(n \leq m)$, the rank of $M_{1}$ is equal to $n+\ell+n \ell$. Therefore, we get (3.3).

\section{Proof of Theorem 2}

As in the proof of Theorem 1, set $F_{\alpha}=F_{\pi}$, where $F_{\alpha}$ is given by (3.1) in Section 3, For a given $C^{r}$ injection $f: N \rightarrow U$, the $C^{r}$ mapping $F_{\alpha} \circ f: N \rightarrow \mathbb{R}^{\ell}$ is given by the same expression as (3.2). Since we have the natural identification $\mathcal{L}\left(\mathbb{R}^{m}, \mathbb{R}^{\ell}\right)=\left(\mathbb{R}^{m}\right)^{\ell}$, in order to prove that there exists a subset $\Sigma$ of $\mathcal{L}\left(\mathbb{R}^{m}, \mathbb{R}^{\ell}\right)$ with Lebesgue measure zero such that for any $\pi \in \mathcal{L}\left(\mathbb{R}^{m}, \mathbb{R}^{\ell}\right)-\Sigma$, and for any $s$ $\left(2 \leq s \leq s_{f}\right)$, the $C^{r}$ mapping $\left(F_{\pi} \circ f\right)^{(s)}: N^{(s)} \rightarrow\left(\mathbb{R}^{\ell}\right)^{s}$ is transverse to $\Delta_{s}$, it is sufficient to prove that there exists a subset $\Sigma$ of $\left(\mathbb{R}^{m}\right)^{\ell}$ with Lebesgue measure zero such that for any $\alpha \in\left(\mathbb{R}^{m}\right)^{\ell}-\Sigma$, and for any $s\left(2 \leq s \leq s_{f}\right)$, the $C^{r}$ mapping $\left(F_{\alpha} \circ f\right)^{(s)}: N^{(s)} \rightarrow\left(\mathbb{R}^{\ell}\right)^{s}$ is transverse to the submanifold $\Delta_{s}$.

Now, let $s$ be a positive integer satisfying $2 \leq s \leq s_{f}$. Let $\Gamma: N^{(s)} \times\left(\mathbb{R}^{m}\right)^{\ell} \rightarrow$ $\left(\mathbb{R}^{\ell}\right)^{s}$ be the $C^{r}$ mapping given by

$$
\Gamma\left(q_{1}, q_{2}, \ldots, q_{s}, \alpha\right)=\left(\left(F_{\alpha} \circ f\right)\left(q_{1}\right),\left(F_{\alpha} \circ f\right)\left(q_{2}\right), \ldots,\left(F_{\alpha} \circ f\right)\left(q_{s}\right)\right) .
$$

Note that from $r>\max \left\{s_{0}, 0\right\}$, we have

$$
\begin{aligned}
r & >\max \{s(n-\ell)+\ell, 0\} \\
& =\max \left\{\operatorname{dim} N^{(s)}-\operatorname{codim} \Delta_{s}, 0\right\}
\end{aligned}
$$

for any positive integer $s\left(2 \leq s \leq s_{f}\right)$. Thus, if for any positive integer $s(2 \leq s \leq$ $s_{f}$ ), the mapping $\Gamma$ is transverse to $\Delta_{s}$, then from Lemma 1, for any positive integer $s\left(2 \leq s \leq s_{f}\right)$, there exists a subset $\Sigma_{s}$ of $\left(\mathbb{R}^{m}\right)^{\ell}$ with Lebesgue measure zero such that for any $\alpha \in\left(\mathbb{R}^{m}\right)^{\ell}-\Sigma_{s}$, the mapping $\Gamma_{\alpha}: N^{(s)} \rightarrow\left(\mathbb{R}^{\ell}\right)^{s}\left(\Gamma_{\alpha}=\left(F_{\alpha} \circ f\right)^{(s)}\right)$ is transverse to $\Delta_{s}$. Then, $\Sigma=\bigcup_{s=2}^{s_{f}} \Sigma_{s}$ is a subset of $\left(\mathbb{R}^{m}\right)^{\ell}$ with Lebesgue measure zero. Thus, for any $\alpha \in\left(\mathbb{R}^{m}\right)^{\ell}-\Sigma$, and for any $s\left(2 \leq s \leq s_{f}\right)$, the $C^{r}$ mapping $\Gamma_{\alpha}: N^{(s)} \rightarrow\left(\mathbb{R}^{\ell}\right)^{s}\left(\Gamma_{\alpha}=\left(F_{\alpha} \circ f\right)^{(s)}\right)$ is transverse to $\Delta_{s}$.

Therefore, for this proof, it is sufficient to prove that for any positive integer $s$ $\left(2 \leq s \leq s_{f}\right)$, if $\Gamma(\widetilde{q}, \widetilde{\alpha}) \in \Delta_{s}\left(\widetilde{q}=\left(\widetilde{q}_{1}, \widetilde{q}_{2}, \ldots, \widetilde{q}_{s}\right)\right)$, then the following holds:

$$
d \Gamma_{(\widetilde{q}, \widetilde{\alpha})}\left(T_{(\widetilde{q}, \widetilde{\alpha})}\left(N^{(s)} \times\left(\mathbb{R}^{m}\right)^{\ell}\right)\right)+T_{\Gamma(\widetilde{q}, \widetilde{\alpha})} \Delta_{s}=T_{\Gamma(\widetilde{q}, \widetilde{\alpha})}\left(\mathbb{R}^{\ell}\right)^{s} .
$$

Let $\left\{\left(U_{\lambda}, \varphi_{\lambda}\right)\right\}_{\lambda \in \Lambda}$ be a coordinate neighborhood system of $N$. There exists a coordinate neighborhood $\left(U_{\widetilde{\lambda}_{1}} \times U_{\widetilde{\lambda}_{2}} \times \cdots \times U_{\widetilde{\lambda}_{s}} \times\left(\mathbb{R}^{m}\right)^{\ell}, \varphi_{\widetilde{\lambda}_{1}} \times \varphi_{\widetilde{\lambda}_{2}} \times \cdots \times \varphi_{\widetilde{\lambda}_{s}} \times i d\right)$ contain$\operatorname{ing}(\widetilde{q}, \widetilde{\alpha})$ of $N^{(s)} \times\left(\mathbb{R}^{m}\right)^{\ell}$, where $i d:\left(\mathbb{R}^{m}\right)^{\ell} \rightarrow\left(\mathbb{R}^{m}\right)^{\ell}$ is the identity mapping, and $\varphi_{\widetilde{\lambda}_{1}} \times \varphi_{\widetilde{\lambda}_{2}} \times \cdots \times \varphi_{\widetilde{\lambda}_{s}} \times i d: U_{\widetilde{\lambda}_{1}} \times U_{\widetilde{\lambda}_{2}} \times \cdots \times U_{\widetilde{\lambda}_{s}} \times\left(\mathbb{R}^{m}\right)^{\ell} \rightarrow\left(\mathbb{R}^{n}\right)^{s} \times\left(\mathbb{R}^{m}\right)^{\ell}$ is defined by $\left(\varphi_{\widetilde{\lambda}_{1}} \times \varphi_{\widetilde{\lambda}_{2}} \times \cdots \times \varphi_{\widetilde{\lambda}_{s}} \times i d\right)\left(q_{1}, q_{2}, \ldots, q_{s}, \alpha\right)=\left(\varphi_{\widetilde{\lambda}_{1}}\left(q_{1}\right), \varphi_{\widetilde{\lambda}_{2}}\left(q_{2}\right), \ldots, \varphi_{\widetilde{\lambda}_{s}}\left(q_{s}\right), i d(\alpha)\right)$. 
Let $t_{i}=\left(t_{i 1}, t_{i 2}, \ldots, t_{i n}\right)$ be a local coordinate around $\varphi_{\widetilde{\lambda}_{i}}\left(\widetilde{q}_{i}\right)(1 \leq i \leq s)$. Then, $\Gamma$ is locally given by the following:

$$
\begin{aligned}
& \Gamma \circ\left(\varphi_{\widetilde{\lambda}_{1}} \times \varphi_{\widetilde{\lambda}_{2}} \times \cdots \times \varphi_{\widetilde{\lambda}_{s}} \times i d\right)^{-1}\left(t_{1}, t_{2}, \ldots, t_{s}, \alpha\right) \\
& =\left(\left(F_{\alpha} \circ f \circ \varphi_{\tilde{\lambda}_{1}}^{-1}\right)\left(t_{1}\right),\left(F_{\alpha} \circ f \circ \varphi_{\tilde{\lambda}_{2}}^{-1}\right)\left(t_{2}\right), \ldots,\left(F_{\alpha} \circ f \circ \varphi_{\tilde{\lambda}_{s}}^{-1}\right)\left(t_{s}\right)\right) \\
& =\left(F_{1} \circ \widetilde{f}\left(t_{1}\right)+\sum_{j=1}^{m} \alpha_{1 j} \tilde{f}_{j}\left(t_{1}\right), F_{2} \circ \widetilde{f}\left(t_{1}\right)+\sum_{j=1}^{m} \alpha_{2 j} \tilde{f}_{j}\left(t_{1}\right), \ldots, F_{\ell} \circ \widetilde{f}\left(t_{1}\right)+\sum_{j=1}^{m} \alpha_{\ell j} \tilde{f}_{j}\left(t_{1}\right),\right. \\
& F_{1} \circ \widetilde{f}\left(t_{2}\right)+\sum_{j=1}^{m} \alpha_{1 j} \tilde{f}_{j}\left(t_{2}\right), F_{2} \circ \widetilde{f}\left(t_{2}\right)+\sum_{j=1}^{m} \alpha_{2 j} \tilde{f}_{j}\left(t_{2}\right), \ldots, F_{\ell} \circ \widetilde{f}\left(t_{2}\right)+\sum_{j=1}^{m} \alpha_{\ell j} \tilde{f}_{j}\left(t_{2}\right), \\
& \text {........, } \\
& \left.F_{1} \circ \widetilde{f}\left(t_{s}\right)+\sum_{j=1}^{m} \alpha_{1 j} \tilde{f}_{j}\left(t_{s}\right), F_{2} \circ \widetilde{f}\left(t_{s}\right)+\sum_{j=1}^{m} \alpha_{2 j} \widetilde{f}_{j}\left(t_{s}\right), \ldots, F_{\ell} \circ \widetilde{f}\left(t_{s}\right)+\sum_{j=1}^{m} \alpha_{\ell j} \tilde{f}_{j}\left(t_{s}\right)\right)
\end{aligned}
$$

where $\tilde{f}\left(t_{i}\right)=\left(\tilde{f}_{1}\left(t_{i}\right), \widetilde{f}_{2}\left(t_{i}\right), \ldots, \widetilde{f}_{m}\left(t_{i}\right)\right)=\left(f_{1} \circ \varphi_{\tilde{\lambda}_{i}}^{-1}\left(t_{i}\right), f_{2} \circ \varphi_{\tilde{\lambda}_{i}}^{-1}\left(t_{i}\right), \ldots, f_{m} \circ\right.$ $\left.\varphi_{\widetilde{\lambda}_{i}}^{-1}\left(t_{i}\right)\right)(1 \leq i \leq s)$. For simplicity, set $t=\left(t_{1}, t_{2}, \ldots, t_{s}\right)$ and $z=\left(\varphi_{\tilde{\lambda}_{1}} \times \varphi_{\tilde{\lambda}_{2}} \times\right.$ $\left.\cdots \times \varphi_{\widetilde{\lambda}_{s}}\right)\left(\widetilde{q}_{1}, \widetilde{q}_{2}, \ldots, \widetilde{q}_{s}\right)$.

The Jacobian matrix of $\Gamma$ at $(\widetilde{q}, \widetilde{\alpha})$ is the following:

$$
J \Gamma_{(\widetilde{q}, \widetilde{\alpha})}=\left(\begin{array}{c|c}
* & B\left(t_{1}\right) \\
* & B\left(t_{2}\right) \\
\vdots & \vdots \\
* & B\left(t_{s}\right)
\end{array}\right)_{(t, \alpha)=(z, \widetilde{\alpha})}
$$

where

$$
\left.B\left(t_{i}\right)=\left(\begin{array}{ccccc}
\mathbf{b}\left(t_{i}\right) & & & 0 & \\
& \mathbf{b}\left(t_{i}\right) & & & \\
0 & & \ddots & & \\
0 & & & & \mathbf{b}\left(t_{i}\right)
\end{array}\right)\right\} \ell \text { rows }
$$

and $\mathbf{b}\left(t_{i}\right)=\left(\widetilde{f}_{1}\left(t_{i}\right), \widetilde{f}_{2}\left(t_{i}\right), \ldots, \widetilde{f}_{m}\left(t_{i}\right)\right)$. By the construction of $T_{\Gamma(\widetilde{q}, \widetilde{\alpha})} \Delta_{s}$, in order to prove (4.1), it is sufficient to prove that the rank of the following matrix $M_{2}$ is equal to $\ell s$ :

$$
M_{2}=\left(\begin{array}{c|c}
E_{\ell} & B\left(t_{1}\right) \\
E_{\ell} & B\left(t_{2}\right) \\
\vdots & \vdots \\
E_{\ell} & B\left(t_{s}\right)
\end{array}\right)_{t=z}
$$


There exists an $\ell s \times \ell s$ regular matrix $Q_{1}$ satisfying

$$
Q_{1} M_{2}=\left(\begin{array}{c|c}
E_{\ell} & B\left(t_{1}\right) \\
0 & B\left(t_{2}\right)-B\left(t_{1}\right) \\
\vdots & \vdots \\
0 & B\left(t_{s}\right)-B\left(t_{1}\right)
\end{array}\right)_{t=z} .
$$

There exists an $(\ell+m \ell) \times(\ell+m \ell)$ regular matrix $Q_{2}$ satisfying

$$
\begin{aligned}
& Q_{1} M_{2} Q_{2}=\left(\begin{array}{c|c}
E_{\ell} & 0 \\
0 & B\left(t_{2}\right)-B\left(t_{1}\right) \\
\vdots & \vdots \\
0 & B\left(t_{s}\right)-B\left(t_{1}\right)
\end{array}\right)_{t=z}
\end{aligned}
$$

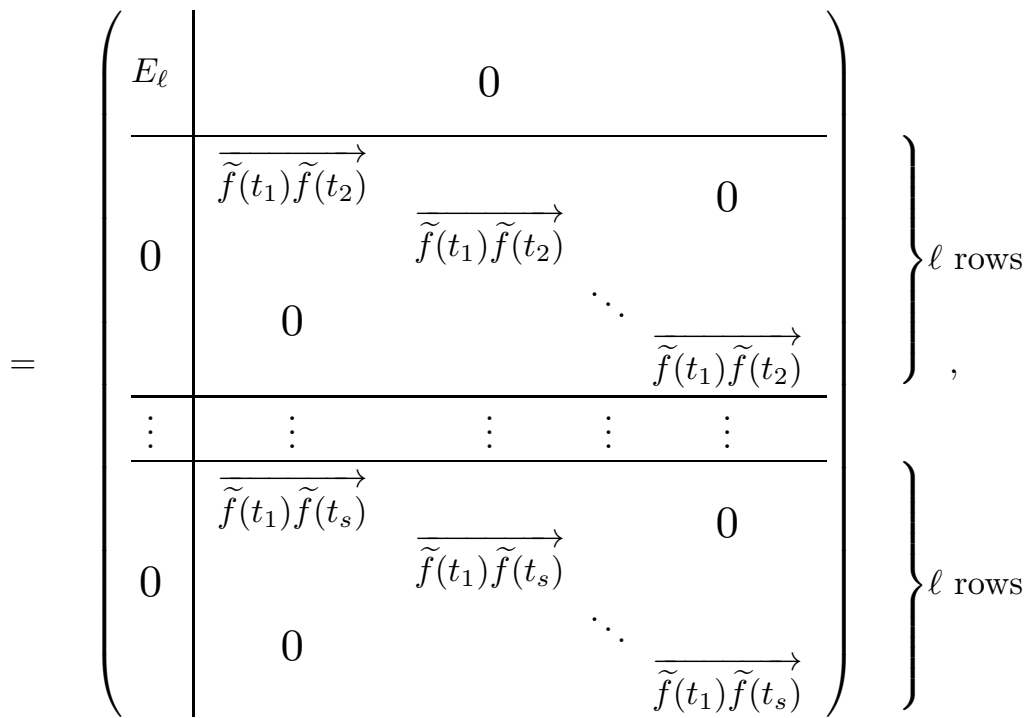

where $\overrightarrow{\widetilde{f}\left(t_{1}\right) \widetilde{f}\left(t_{i}\right)}=\left(\widetilde{f}_{1}\left(t_{i}\right)-\widetilde{f}_{1}\left(t_{1}\right), \widetilde{f}_{2}\left(t_{i}\right)-\widetilde{f}_{2}\left(t_{1}\right), \ldots, \widetilde{f}_{m}\left(t_{i}\right)-\widetilde{f}_{m}\left(t_{1}\right)\right)(2 \leq i \leq s)$ and $t=z$. From $s-1 \leq s_{f}-1$ and the definition of $s_{f}$, we have

$$
\operatorname{dim} \sum_{i=2}^{s} \mathbb{R} \overrightarrow{\widetilde{f}\left(t_{1}\right) \widetilde{f}\left(t_{i}\right)}=s-1
$$

where $t=z$. Hence, by the construction of $Q_{1} M_{2} Q_{2}$ and $s-1 \leq m$, the rank of $Q_{1} M_{2} Q_{2}$ is equal to $\ell s$. Therefore, the rank of $M_{2}$ must be equal to $\ell s$. Hence, we get (4.1). Therefore, there exists a subset $\Sigma$ of $\mathcal{L}\left(\mathbb{R}^{m}, \mathbb{R}^{\ell}\right)$ with Lebesgue measure zero such that for any $\pi \in \mathcal{L}\left(\mathbb{R}^{m}, \mathbb{R}^{\ell}\right)-\Sigma$, and for any $s\left(2 \leq s \leq s_{f}\right)$, the $C^{r}$ mapping $\left(F_{\pi} \circ f\right)^{(s)}: N^{(s)} \rightarrow\left(\mathbb{R}^{\ell}\right)^{s}$ is transverse to $\Delta_{s}$.

Moreover, suppose that the $C^{r}$ mapping $F_{\pi}$ satisfies that $\left|F_{\pi}^{-1}(y)\right| \leq s_{f}$ for any $y \in \mathbb{R}^{\ell}$. Since $f: N \rightarrow \mathbb{R}^{m}$ is injective, it follows that $\left|\left(F_{\pi} \circ f\right)^{-1}(y)\right| \leq$ $s_{f}$ for any $y \in \mathbb{R}^{\ell}$. Thus, for any positive integer $s$ with $s \geq s_{f}+1$, we have $\left(F_{\pi} \circ f\right)^{(s)}\left(N^{(s)}\right) \bigcap \Delta_{s}=\emptyset$. Namely, for any positive integer $s$ with $s \geq s_{f}+1$, the $C^{r}$ mapping $\left(F_{\pi} \circ f\right)^{(s)}$ is transverse to $\Delta_{s}$. Hence, $F_{\pi} \circ f: N \rightarrow \mathbb{R}^{\ell}$ is a $C^{r}$ mapping with normal crossings. 


\section{Applications of Theorems 1 and 2}

In Section 5.1 (resp., Section 5.2), applications of Theorem 1 (resp., Theorem 2) are stated and proved. In Section [5.2, applications obtained by combining Theorems 1 and 2 are also given.

5.1. Applications of Theorem 1, A $C^{2}$ function $g: N \rightarrow \mathbb{R}$ is called a Morse function if all of the critical points of $g$ are nondegenerate, where $N$ is a $C^{2}$ manifold of dimension $n$ (for details on Morse functions, see for instance, [1, p. 63]). In the case of $(n, \ell)=(n, 1)$, we have the following.

Corollary 1. Let $f$ be a $C^{2}$ immersion of $N$ into an open subset $U$ of $\mathbb{R}^{m}$, where $N$ is a $C^{2}$ manifold of dimension n. Let $F: U \rightarrow \mathbb{R}$ be a $C^{2}$ function. Then, there exists a subset $\Sigma$ of $\mathcal{L}\left(\mathbb{R}^{m}, \mathbb{R}\right)$ with Lebesgue measure zero such that for any $\pi \in \mathcal{L}\left(\mathbb{R}^{m}, \mathbb{R}\right)-\Sigma$, the $C^{2}$ function $F_{\pi} \circ f: N \rightarrow \mathbb{R}$ is a Morse function.

Proof. We have $\operatorname{dim} N-\operatorname{codim} \Sigma^{1}(N, \mathbb{R})=0$. Therefore, from Theorem [1 there exists a subset $\Sigma$ with Lebesgue measure zero of $\mathcal{L}\left(\mathbb{R}^{m}, \mathbb{R}\right)$ such that for any $\pi \in \mathcal{L}\left(\mathbb{R}^{m}, \mathbb{R}\right)-\Sigma$, the mapping $j^{1}\left(F_{\pi} \circ f\right): N \rightarrow J^{1}(N, \mathbb{R})$ is transverse to $\Sigma^{1}(N, \mathbb{R})$. Therefore, if $q \in N$ is a critical point of the function $F_{\pi} \circ f$, then the point $q$ is nondegenerate.

In the case of $\ell \geq 2 n$, we have the following.

Corollary 2. Let $f$ be a $C^{2}$ immersion of $N$ into an open subset $U$ of $\mathbb{R}^{m}$, where $N$ is a $C^{2}$ manifold of dimension $n$. Let $F: U \rightarrow \mathbb{R}^{\ell}$ be a $C^{2}$ mapping $(\ell \geq 2 n)$. Then, there exists a subset $\Sigma$ of $\mathcal{L}\left(\mathbb{R}^{m}, \mathbb{R}^{\ell}\right)$ with Lebesgue measure zero such that for any $\pi \in \mathcal{L}\left(\mathbb{R}^{m}, \mathbb{R}^{\ell}\right)-\Sigma$, the mapping $F_{\pi} \circ f: N \rightarrow \mathbb{R}^{\ell}$ is a $C^{2}$ immersion.

Proof. It is clearly seen that $F_{\pi} \circ f: N \rightarrow \mathbb{R}^{\ell}$ is an immersion if and only if $j^{1}\left(F_{\pi} \circ f\right)(N) \cap \bigcup_{k=1}^{n} \Sigma^{k}\left(N, \mathbb{R}^{\ell}\right)=\emptyset$. From $\ell \geq 2 n$, for any positive integer $k$ $(1 \leq k \leq n)$, we have

$$
\operatorname{dim} N-\operatorname{codim} \Sigma^{k}\left(N, \mathbb{R}^{\ell}\right)=n-k(\ell-n+k) \leq 0 .
$$

Thus, for any positive integer $k(1 \leq k \leq n)$, from Theorem 1, there exists a subset $\widetilde{\Sigma}_{k}$ of $\mathcal{L}\left(\mathbb{R}^{m}, \mathbb{R}^{\ell}\right)$ with Lebesgue measure zero such that for any $\pi \in \mathcal{L}\left(\mathbb{R}^{m}, \mathbb{R}^{\ell}\right)-\widetilde{\Sigma}_{k}$, the mapping $j^{1}\left(F_{\pi} \circ f\right): N \rightarrow J^{1}\left(N, \mathbb{R}^{\ell}\right)$ is transverse to $\Sigma^{k}\left(N, \mathbb{R}^{\ell}\right)$. Set $\Sigma=$ $\bigcup_{k=1}^{n} \widetilde{\Sigma}_{k}$. Note that $\Sigma$ has Lebesgue measure zero. Let $\pi \in \mathcal{L}\left(\mathbb{R}^{m}, \mathbb{R}^{\ell}\right)-\Sigma$ be an arbitrary element. Then, suppose that there exists a point $q \in N$ and a positive integer $k(1 \leq k \leq n)$ such that $j^{1}\left(F_{\pi} \circ f\right)(q) \in \Sigma^{k}\left(N, \mathbb{R}^{\ell}\right)$. Since $j^{1}\left(F_{\pi} \circ f\right)$ is transverse to $\Sigma^{k}\left(N, \mathbb{R}^{\ell}\right)$, we have the following:

$$
d\left(j^{1}\left(F_{\pi} \circ f\right)\right)_{q}\left(T_{q} N\right)+T_{j^{1}\left(F_{\pi} \circ f\right)(q)} \Sigma^{k}\left(N, \mathbb{R}^{\ell}\right)=T_{j^{1}\left(F_{\pi} \circ f\right)(q)} J^{1}\left(N, \mathbb{R}^{\ell}\right) .
$$

Hence, we have

$$
\begin{aligned}
\operatorname{dim} d\left(j^{1}\left(F_{\pi} \circ f\right)\right)_{q}\left(T_{q} N\right) & \geq \operatorname{dim} T_{j^{1}\left(F_{\pi} \circ f\right)(q)} J^{1}\left(N, \mathbb{R}^{\ell}\right)-\operatorname{dim} T_{j^{1}\left(F_{\pi} \circ f\right)(q)} \Sigma^{k}\left(N, \mathbb{R}^{\ell}\right) \\
& =\operatorname{codim} T_{j^{1}\left(F_{\pi} \circ f\right)(q)} \Sigma^{k}\left(N, \mathbb{R}^{\ell}\right) .
\end{aligned}
$$

Thus, we get $n \geq k(\ell-n+k)$. This contradicts the assumption $\ell \geq 2 n$. Therefore, we get $j^{1}\left(F_{\pi} \circ f\right)(N) \bigcap \bigcup_{k=1}^{n} \Sigma^{k}\left(N, \mathbb{R}^{\ell}\right)=\emptyset$.

A $C^{1}$ mapping $g: N \rightarrow \mathbb{R}^{\ell}$ has singular points of corank at most $k$ if $\sup \left\{\operatorname{corank} d g_{q} \mid q \in N\right\} \leq k$, 
where corank $d g_{q}=\min \{n, \ell\}-\operatorname{rank} d g_{q}$.

Corollary 3. Let $f$ be a $C^{r}$ immersion of $N$ into an open subset $U$ of $\mathbb{R}^{m}$, where $N$ is a $C^{r}$ manifold of dimension $n$. Let $F: U \rightarrow \mathbb{R}^{\ell}$ be a $C^{r}$ mapping. Let $k_{0}$ be the maximum integer satisfying $\left(n-v+k_{0}\right)\left(\ell-v+k_{0}\right) \leq n(v=\min \{n, \ell\})$. If

$$
r>\max \left\{\operatorname{dim} N-\operatorname{codim} \Sigma^{1}\left(N, \mathbb{R}^{\ell}\right), 0\right\}+1,
$$

then there exists a subset $\Sigma$ of $\mathcal{L}\left(\mathbb{R}^{m}, \mathbb{R}^{\ell}\right)$ with Lebesgue measure zero such that for any $\pi \in \mathcal{L}\left(\mathbb{R}^{m}, \mathbb{R}^{\ell}\right)-\Sigma$, the $C^{r}$ mapping $F_{\pi} \circ f: N \rightarrow \mathbb{R}^{\ell}$ has singular points of corank at most $k_{0}$.

Proof. For any positive integer $k(1 \leq k \leq v)$, we have

$$
\begin{aligned}
r & >\max \left\{\operatorname{dim} N-\operatorname{codim} \Sigma^{1}\left(N, \mathbb{R}^{\ell}\right), 0\right\}+1 \\
& \geq \max \left\{\operatorname{dim} N-\operatorname{codim} \Sigma^{k}\left(N, \mathbb{R}^{\ell}\right), 0\right\}+1 .
\end{aligned}
$$

From Theorem 1, for any positive integer $k$ satisfying $1 \leq k \leq v$, there exists a subset $\widetilde{\Sigma}_{k}$ of $\mathcal{L}\left(\mathbb{R}^{m}, \mathbb{R}^{\ell}\right)$ with Lebesgue measure zero such that for any $\pi \in \mathcal{L}\left(\mathbb{R}^{m}, \mathbb{R}^{\ell}\right)-\widetilde{\Sigma}_{k}$, the mapping $j^{1}\left(F_{\pi} \circ f\right): N \rightarrow J^{1}\left(N, \mathbb{R}^{\ell}\right)$ is transverse to $\Sigma^{k}\left(N, \mathbb{R}^{\ell}\right)$. Then, $\Sigma=\bigcup_{k=1}^{v} \widetilde{\Sigma}_{k}$ has Lebesgue measure zero. Hence, there exists a subset $\Sigma$ of $\mathcal{L}\left(\mathbb{R}^{m}, \mathbb{R}^{\ell}\right)$ with Lebesgue measure zero such that for any $\pi \in \mathcal{L}\left(\mathbb{R}^{m}, \mathbb{R}^{\ell}\right)-\Sigma$, the mapping $j^{1}\left(F_{\pi} \circ f\right): N \rightarrow J^{1}\left(N, \mathbb{R}^{\ell}\right)$ is transverse to $\Sigma^{k}\left(N, \mathbb{R}^{\ell}\right)$ for any positive integer $k$ satisfying $1 \leq k \leq v$.

In the case of $\ell=1$, we have $k_{0}=1$. Thus, in this case, the assertion clearly holds.

Now, we will consider the case of $\ell \geq 2$. In this case, note that $k_{0}+1 \leq v$. Indeed, suppose that $v \leq k_{0}$. Then, by $\left(n-v+k_{0}\right)\left(\ell-v+k_{0}\right) \leq n$, we get $n \ell \leq n$. This contradicts the assumption $\ell \geq 2$. For the proof of Corollary 3 it is sufficient to show that the mapping $j^{1}\left(F_{\pi} \circ f\right): N \rightarrow J^{1}\left(N, \mathbb{R}^{\ell}\right)$ satisfies that $j^{1}\left(F_{\pi} \circ f\right)(N) \bigcap \Sigma^{k}\left(N, \mathbb{R}^{\ell}\right)=\emptyset$ for any positive integer $k$ satisfying $k_{0}+1 \leq k \leq v$. Suppose that there exist a positive integer $k\left(k_{0}+1 \leq k \leq v\right)$ and a point $q \in N$ such that $j^{1}\left(F_{\pi} \circ f\right)(q) \in \Sigma^{k}\left(N, \mathbb{R}^{\ell}\right)$. Since the mapping $j^{1}\left(F_{\pi} \circ f\right): N \rightarrow J^{1}\left(N, \mathbb{R}^{\ell}\right)$ is transverse to $\Sigma^{k}\left(N, \mathbb{R}^{\ell}\right)$ at the point $q$, the following holds:

$$
d\left(j^{1}\left(F_{\pi} \circ f\right)\right)_{q}\left(T_{q} N\right)+T_{j^{1}\left(F_{\pi} \circ f\right)(q)} \Sigma^{k}\left(N, \mathbb{R}^{\ell}\right)=T_{j^{1}\left(F_{\pi} \circ f\right)(q)} J^{1}\left(N, \mathbb{R}^{\ell}\right) .
$$

Hence, we have

$$
\begin{aligned}
& \operatorname{dim} d\left(j^{1}\left(F_{\pi} \circ f\right)\right)_{q}\left(T_{q} N\right) \\
\geq & \operatorname{dim} T_{j^{1}\left(F_{\pi} \circ f\right)(q)} J^{1}\left(N, \mathbb{R}^{\ell}\right)-\operatorname{dim} T_{j^{1}\left(F_{\pi} \circ f\right)(q)} \Sigma^{k}\left(N, \mathbb{R}^{\ell}\right) \\
= & \operatorname{codim} T_{j^{1}\left(F_{\pi} \circ f\right)(q)} \Sigma^{k}\left(N, \mathbb{R}^{\ell}\right) .
\end{aligned}
$$

Thus, we get $n \geq(n-v+k)(\ell-v+k)$. Since the given integer $k_{0}$ is the maximum integer satisfying $n \geq\left(n-v+k_{0}\right)\left(\ell-v+k_{0}\right)$, it follows that $k \leq k_{0}$. This contradicts the assumption $k_{0}+1 \leq k$.

\subsection{Applications of Theorem 2 ,}

Corollary 4. Let $f$ be a $C^{r}$ injection of $N$ into an open subset $U$ of $\mathbb{R}^{m}$, where $N$ is a $C^{r}$ manifold of dimension $n$. Let $F: U \rightarrow \mathbb{R}^{\ell}$ be a $C^{r}$ mapping. If

$$
\left(s_{f}-1\right) \ell>n s_{f} \text { and } r>\max \{2 n-\ell, 0\},
$$


then there exists a subset $\Sigma$ of $\mathcal{L}\left(\mathbb{R}^{m}, \mathbb{R}^{\ell}\right)$ with Lebesgue measure zero such that for any $\pi \in \mathcal{L}\left(\mathbb{R}^{m}, \mathbb{R}^{\ell}\right)-\Sigma, F_{\pi} \circ f: N \rightarrow \mathbb{R}^{\ell}$ is a $C^{r}$ mapping with normal crossings satisfying $\left(F_{\pi} \circ f\right)^{\left(s_{f}\right)}\left(N^{\left(s_{f}\right)}\right) \bigcap \Delta_{s_{f}}=\emptyset$.

Proof. $\quad$ From $\left(s_{f}-1\right) \ell>n s_{f}$, we have $n-\ell<0$. Thus, we get

$$
\begin{aligned}
s_{0} & =\max \left\{s(n-\ell)+\ell \mid 2 \leq s \leq s_{f}\right\} \\
& =2 n-\ell .
\end{aligned}
$$

Hence, note that $r>\max \left\{s_{0}, 0\right\}$. From Theorem 2, there exists a subset $\Sigma$ of $\mathcal{L}\left(\mathbb{R}^{m}, \mathbb{R}^{\ell}\right)$ with Lebesgue measure zero such that for any $\pi \in \mathcal{L}\left(\mathbb{R}^{m}, \mathbb{R}^{\ell}\right)-\Sigma$, and for any $s\left(2 \leq s \leq s_{f}\right)$, the mapping $\left(F_{\pi} \circ f\right)^{(s)}: N^{(s)} \rightarrow\left(\mathbb{R}^{\ell}\right)^{s}$ is transverse to $\Delta_{s}$. Therefore, for this proof, it is sufficient to prove that for any $\pi \in \mathcal{L}\left(\mathbb{R}^{m}, \mathbb{R}^{\ell}\right)-\Sigma$, the mapping $\left(F_{\pi} \circ f\right)^{\left(s_{f}\right)}$ satisfies that $\left(F_{\pi} \circ f\right)^{\left(s_{f}\right)}\left(N^{\left(s_{f}\right)}\right) \bigcap \Delta_{s_{f}}=\emptyset$.

Suppose that there exists an element $\pi \in \mathcal{L}\left(\mathbb{R}^{m}, \mathbb{R}^{\ell}\right)-\Sigma$ such that there exists a point $q \in N^{\left(s_{f}\right)}$ satisfying $\left(F_{\pi} \circ f\right)^{\left(s_{f}\right)}(q) \in \Delta_{s_{f}}$. Since $\left(F_{\pi} \circ f\right)^{\left(s_{f}\right)}$ is transverse to $\Delta_{s_{f}}$, we have the following:

$$
d\left(\left(F_{\pi} \circ f\right)^{\left(s_{f}\right)}\right)_{q}\left(T_{q} N^{\left(s_{f}\right)}\right)+T_{\left(F_{\pi} \circ f\right)^{\left(s_{f}\right)}(q)} \Delta_{s_{f}}=T_{\left(F_{\pi} \circ f\right)^{\left(s_{f}\right)}(q)}\left(\mathbb{R}^{\ell}\right)^{s_{f}} .
$$

Thus, we get

$$
\begin{aligned}
& \operatorname{dim} d\left(\left(F_{\pi} \circ f\right)^{\left(s_{f}\right)}\right)_{q}\left(T_{q} N^{\left(s_{f}\right)}\right) \\
\geq & \operatorname{dim} T_{\left(F_{\pi} \circ f\right)^{\left(s_{f}\right)}(q)}\left(\mathbb{R}^{\ell}\right)^{s_{f}}-\operatorname{dim} T_{\left(F_{\pi} \circ f\right)^{\left(s_{f}\right)}(q)} \Delta_{s_{f}} \\
= & \operatorname{codim} T_{\left(F_{\pi} \circ f\right)^{\left(s_{f}\right)}(q)} \Delta_{s_{f}} .
\end{aligned}
$$

Hence, we have $n s_{f} \geq\left(s_{f}-1\right) \ell$. This contradicts the assumption $\left(s_{f}-1\right) \ell>n s_{f}$.

In the case of $\ell>2 n$, we have the following.

Corollary 5. Let $f$ be a $C^{1}$ injection of $N$ into an open subset $U$ of $\mathbb{R}^{m}$, where $N$ is a $C^{1}$ manifold of dimension $n$. Let $F: U \rightarrow \mathbb{R}^{\ell}$ be a $C^{1}$ mapping. If $\ell>2 n$, then there exists a subset $\Sigma$ of $\mathcal{L}\left(\mathbb{R}^{m}, \mathbb{R}^{\ell}\right)$ with Lebesgue measure zero such that for any $\pi \in \mathcal{L}\left(\mathbb{R}^{m}, \mathbb{R}^{\ell}\right)-\Sigma$, the $C^{1}$ mapping $F_{\pi} \circ f: N \rightarrow \mathbb{R}^{\ell}$ is injective.

Proof. $\quad$ Since $s_{f} \geq 2$ and $\ell>2 n$, it is easily seen that the dimension pair $(n, \ell)$ satisfies the assumption $\left(s_{f}-1\right) \ell>n s_{f}$ of Corollary 4 . Indeed, from $\ell>2 n$, we get $\left(s_{f}-1\right) \ell>2 n\left(s_{f}-1\right)$. From $s_{f} \geq 2$, it follows that $2 n\left(s_{f}-1\right) \geq n s_{f}$.

Since $\max \{2 n-\ell, 0\}=0$, from Corollary 4 there exists a subset $\Sigma$ of $\mathcal{L}\left(\mathbb{R}^{m}, \mathbb{R}^{\ell}\right)$ with Lebesgue measure zero such that for any $\pi \in \mathcal{L}\left(\mathbb{R}^{m}, \mathbb{R}^{\ell}\right)-\Sigma$, the mapping $\left(F_{\pi} \circ f\right)^{(2)}: N^{(2)} \rightarrow\left(\mathbb{R}^{\ell}\right)^{2}$ is transverse to $\Delta_{2}$. For this proof, it is sufficient to prove that the mapping $\left(F_{\pi} \circ f\right)^{(2)}$ satisfies that $\left(F_{\pi} \circ f\right)^{(2)}\left(N^{(2)}\right) \bigcap \Delta_{2}=\emptyset$.

Suppose that there exists a point $q \in N^{(2)}$ such that $\left(F_{\pi} \circ f\right)^{(2)}(q) \in \Delta_{2}$. Then, we get the following:

$$
d\left(\left(F_{\pi} \circ f\right)^{(2)}\right)_{q}\left(T_{q} N^{(2)}\right)+T_{\left(F_{\pi} \circ f\right)^{(2)}(q)} \Delta_{2}=T_{\left(F_{\pi} \circ f\right)^{(2)}(q)}\left(\mathbb{R}^{\ell}\right)^{2} .
$$

Thus, we have

$$
\begin{aligned}
& \operatorname{dim} d\left(\left(F_{\pi} \circ f\right)^{(2)}\right)_{q}\left(T_{q} N^{(2)}\right) \\
\geq & \operatorname{dim} T_{\left(F_{\pi} \circ f\right)^{(2)}(q)}\left(\mathbb{R}^{\ell}\right)^{2}-\operatorname{dim} T_{\left(F_{\pi} \circ f\right)^{(2)}(q)} \Delta_{2} \\
= & \operatorname{codim} T_{\left(F_{\pi} \circ f\right)^{(2)}(q)} \Delta_{2} .
\end{aligned}
$$


Hence, we have $2 n \geq \ell$. This contradicts the assumption $\ell>2 n$.

By combining Corollaries 2 and 5 , we have the following.

Corollary 6. Let $f$ be an injective immersion of $N$ into an open subset $U$ of $\mathbb{R}^{m}$, where $N$ is a $C^{2}$ manifold of dimension $n$ and $f$ is of class $C^{2}$. Let $F: U \rightarrow \mathbb{R}^{\ell}$ be a $C^{2}$ mapping. If $\ell>2 n$, then there exists a subset $\Sigma$ of $\mathcal{L}\left(\mathbb{R}^{m}, \mathbb{R}^{\ell}\right)$ with Lebesgue measure zero such that for any $\pi \in \mathcal{L}\left(\mathbb{R}^{m}, \mathbb{R}^{\ell}\right)-\Sigma$, the $C^{2}$ mapping $F_{\pi} \circ f: N \rightarrow \mathbb{R}^{\ell}$ is an injective immersion.

From Corollary [6] we get the following.

Corollary 7. Let $N$ be a compact $C^{2}$ manifold of dimension $n$. Let $f$ be a $C^{2}$ embedding of $N$ into an open subset $U$ of $\mathbb{R}^{m}$. Let $F: U \rightarrow \mathbb{R}^{\ell}$ be a $C^{2}$ mapping. If $\ell>2 n$, then there exists a subset $\Sigma$ of $\mathcal{L}\left(\mathbb{R}^{m}, \mathbb{R}^{\ell}\right)$ with Lebesgue measure zero such that for any $\pi \in \mathcal{L}\left(\mathbb{R}^{m}, \mathbb{R}^{\ell}\right)-\Sigma$, the $C^{2}$ mapping $F_{\pi} \circ f: N \rightarrow \mathbb{R}^{\ell}$ is an embedding.

\section{Proof of Lemma 1}

6.1. Preliminaries for the proof of Lemma 1, Let $N$ and $P$ be $C^{r}$ manifolds, and let $g: N \rightarrow P$ be a $C^{1}$ mapping $(r \geq 1)$. A point $x \in N$ is called a critical point of $g$ if it is not a regular point, i.e., the rank of $d g_{x}$ is less than the dimension of $P$. We say that a point $y \in P$ is a critical value if it is the image of a critical point. A point $y \in P$ is called a regular value if it is not a critical value. The following is Sard's theorem.

Theorem 3 ([]. $)$. If $N$ and $P$ are $C^{r}$ manifolds, $g: N \rightarrow P$ is a $C^{r}$ mapping, and $r>\max \{\operatorname{dim} N-\operatorname{dim} P, 0\}$, then the set of critical values of $g$ has Lebesgue measure zero.

6.2. Proof of Lemma 1, In this proof, by $\pi: N \times A \rightarrow A$, we denote the natural projection defined by $\pi(x, a)=a$.

Since $\Gamma$ is transverse to $Z$, the set $\Gamma^{-1}(Z)$ is a $C^{r}$ submanifold of $N \times A$ satisfying

$$
\operatorname{dim} N+\operatorname{dim} A-\operatorname{dim} \Gamma^{-1}(Z)=\operatorname{dim} P-\operatorname{dim} Z .
$$

Firstly, suppose that $\operatorname{dim} \Gamma^{-1}(Z)=0$. Then, since $\Gamma^{-1}(Z)$ is a countable set, $\pi\left(\Gamma^{-1}(Z)\right)$ has Lebesgue measure zero in $A$. It is clearly seen that for any $a \in$ $A-\pi\left(\Gamma^{-1}(Z)\right)$, the mapping $\Gamma_{a}$ is transverse to $Z$.

Finally, we will consider the case $\operatorname{dim} \Gamma^{-1}(Z)>0$. It is not hard to see that if $a \in A$ is a regular value of $\left.\pi\right|_{\Gamma^{-1}(Z)}$, then $\Gamma_{a}$ is transverse to $Z$, where $\left.\pi\right|_{\Gamma^{-1}(Z)}$ is the restriction of $\pi$ to $\Gamma^{-1}(Z)$. Let $\Sigma$ be the set of critical values of $\left.\pi\right|_{\Gamma^{-1}(Z)}$. From $r>$ $\max \{\operatorname{dim} N+\operatorname{dim} Z-\operatorname{dim} P, 0\}$ and (1), we have $r>\max \left\{\operatorname{dim} \Gamma^{-1}(Z)-\operatorname{dim} A, 0\right\}$. From Theorem 3, $\Sigma$ has Lebesgue measure zero in $A$. Therefore, if $a \in A-\Sigma$, then $\Gamma_{a}$ is transverse to $Z$.

\section{ACKNOWLEDGEMENTS}

The author is most grateful to the anonymous reviewer for carefully reading the first manuscript of this paper and for giving invaluable suggestions. The author was supported by JSPS KAKENHI Grant Number 16J06911. 


\section{REFERENCES}

[1] M. Golubitsky and V. Guillemin, Stable mappings and their singularities, Graduate Texts in Mathematics 14, Springer, New York, 1973.

[2] S. Ichiki, Composing generic linearly perturbed mappings and immersions/injections, to appear in J. Math. Soc. Japan, available from arXiv:1612.01100.

[3] S. Ichiki, Generic linear perturbations, to appear in Proc. Amer. Math. Soc., available from arXiv:1607.03220

[4] J. N. Mather, Stability of $C^{\infty}$ mappings V. The nice dimensions, Lecture Notes in Math., 192 (1971), 207-253.

[5] J. N. Mather, Generic projections, Ann. of Math., (2), 98 (1973), 226-245.

[6] A. Sard, The measure of the critical values of differentiable maps, Bull. Amer. Math. Soc. 48 (1942), 883-890.

Graduate School of Environment and Information Sciences, Yokohama National UniVERSITY, YOKOHAMA 240-8501, JAPAN

E-mail address: ichiki-shunsuke-jb@ynu.jp 